\title{
Unveiling the links between peptide identification and differential analysis FDR controls by means of a practical introduction to knockoff filters
}

\author{
Lucas Etourneau $^{1,2}$, Nelle Varoquaux ${ }^{1}$, and Thomas Burger ${ }^{2}$ \\ ${ }^{1}$ Univ. Grenoble Alpes, CNRS, Grenoble INP, TIMC-IMAG, 38000 Grenoble \\ ${ }^{2}$ Univ. Grenoble Alpes, CNRS, CEA, Inserm, ProFI FR2048, Grenoble, France
}

\begin{abstract}
In proteomic differential analysis, FDR control is often performed through a multiple test correction (i.e., the adjustment of the original p-values). In this protocol, we apply a recent and alternative method, based on so-called knockoff filters. It shares interesting conceptual similarities with the target-decoy competition procedure, classically used in proteomics for FDR control at peptide identification. To provide practitioners with a unified understanding of FDR control in proteomics, we apply the knockoff procedure on real and simulated quantitative datasets. Leveraging these comparisons, we propose to adapt the knockoff procedure to better fit the specificities of quantitive proteomic data (mainly very few samples). Performances of knockoff procedure are compared with those of the classical Benjamini-Hochberg procedure, hereby shedding a new light on the strengths and weaknesses of target-decoy competition.
\end{abstract}

\section{Introduction}

Controlling the false discovery rate (FDR) is a well-established practice in most -omic approaches, as it answers a pervasive question: Considering quantitative measurements for many covariates (be they genes, transcripts, metabolites, or proteins) in a set of samples split in at least two different biological conditions, how can we shortlist some differentially expressed ones, while controlling the risk of false positives (i.e. wrongly selected covariates due to their looking differentially expressed while they are not)? To cope with this, the most commonly used procedure is without a doubt the Benjamini-Hochberg one (BH) [2]. However, due to its large field of application, FDR control has focused a lot of additional efforts in biostatistics, and many authors have proposed to improve upon BH FDR control $[3,8]$, or have proposed alternative frameworks to do so $[1,6,20]$.

In the specific case of proteomics, FDR control is not only used in the aforementioned biomarker selection problem. It is also an essential quality control metric when matching experimental fragmentation spectra onto in silico spectra (i.e., derived from reference database of protein sequences). However, for historical reasons, the associated FDR control is not performed using classical tools from 
biostatistics. On the contrary, a rather empirical approach termed target-decoy [9] is almost exclusively used. It consists in searching two databases: the first one, referred to as target, containing the genuine protein sequences, and another one, referred to as decoy, containing artefactual sequences. Under the assumption that target mismatches and decoy matches are equally likely, the number of decoy matches can be used to estimate the number of target mismatches, thus opening the door to FDR control.

For a long time, FDR control for peptide identification and for protein differential analysis have been considered as largely independent. However, theoretical connections exist: Notably, it has long been established [16] that if target and decoy databases are searched independently, then the procedure is broadly equivalent to relying on empirical null theory to estimate the FDR in a BH-related way [8]. More recently, it has been shown ([7] that $\mathrm{BH}$ procedure could be a user-friendly and computationally attractive alternative to target decoy competition (TDC) (see Note 1). However, recent developments in theoretical biostatistics have made the links between both approaches to FDR control even tighter. Notably, the authors of [1] have proposed to tackle the biomarker research FDR control using an algorithmic procedure akin to that of TDC. Broadly, this novel approach, denoted as "knockoff-filter," works as follows. First, knockoff variables are simulated to be as independent as possible from conditions of samples, but yet preserve the covariance structure of the original variables (see Note 2). Second, a competition is organized between each original variable and its associated knockoff. Third, the proportion of retained knockoffs is used to estimate the proportion of wrongly selected original covariates (see Table 1 for a more detailed comparison with TDC). Conversely, authors have recently leverage the theory underlying knockoff filters to propose improved TDC strategies (see [10]).

Overall, the framework of knockoff filters is particularly insightful to provide a global understanding of FDR control in proteomics and the purpose of this protocol is to root such unified view on empirical comparisons using both real and simulated data. Interestingly, the results of these comparisons are compliant with empirical knowledge about the various strengths and weaknesses classically associated to each FDR control method.

\section{Notations}

We first start by reviewing commonly used yet conflicting notations in biostatistics and proteomics.

\subsection{Classical notations in biostatistics}

In biostatistics, the false discovery rate (FDR) and the false discovery proportion (FDP) are distinct notions. The FDP corresponds to what was classically and informally referred to as the "true FDR" in proteomics, i.e., the exact proportion of false positives among the proteins that passed the user-defined selection threshold, and therefore deemed as differentially abundant. Of course, except for benchmark artificial or simulated datasets, this quantity is unknown in practice.

The FDR reads as FDR $=\mathbb{E}[$ FDP $]$, where $\mathbb{E}$ stands for the expectation, which broadly amounts to the long run average of the FDP on an infinite number of related experiments subject to stochastic fluctuations. This quantity is also unknown but it 


\begin{tabular}{|l|l|}
\hline Target-Decoy Competition & $\begin{array}{l}\text { Knockoff filter (2nd order approxi- } \\
\text { mation) }\end{array}$ \\
\hline $\begin{array}{l}\text { 1. Construct peptide decoys such that } \\
\text { decoy PSMs have same score distribu- } \\
\text { tion than erroneous target PSMs }\end{array}$ & $\begin{array}{l}\text { 1. For each protein, generate knockoff } \\
\text { abundances with same mean and corre- } \\
\text { lation matrices as original abundances. }\end{array}$ \\
\hline $\begin{array}{l}\text { 2. For each real spectrum obtained, find } \\
\text { the best match among all targets and } \\
\text { decoys, and retain its score. }\end{array}$ & $\begin{array}{l}\text { 2. For each protein, compute a score } \\
\text { describing whether the original abun- } \\
\text { dances vector or its knockoff best pre- } \\
\text { dicts the condition. }\end{array}$ \\
\hline $\begin{array}{l}\text { 3. The number of selected PSMs from } \\
\text { decoys at a given cutoff enables to esti- } \\
\text { mate the FDR on selected target PSMs. }\end{array}$ & $\begin{array}{l}\text { 3. The number of selected knockoffs } \\
\text { at a given cutoff enables to estimate } \\
\text { the FDR on selected original proteins } \\
\text { deemed differentially abundant. }\end{array}$ \\
\hline
\end{tabular}

Table 1: Comparison of the target-decoy and knockoff filter procedures for FDR control. (PSM stands for Peptide-Spectrum Match).

is much easier to estimate, and such estimate is classically noted $\widehat{\text { FDR }}$. Estimating the FDR is insightful, but unfortunately, not always sufficient [14]. An unbiased FDR estimate is expected to provide a value closed to $\mathbb{E}[$ FDP $]$. However, on a given dataset, this value may be larger or smaller than the FDP. While a slightly too large estimate implies a conservative behavior (there will be less false positives than expected among the shortlisted biomarkers), a too small FDR implies a too liberal quality control and subsequent risks in post-proteomics experiments.

To cope with weaknesses of FDR estimation, FDR control procedures have been developed: they rely on more conservative assumptions that yield slightly lesser selected discoveries at a given cut-off parameter. If we note as $\widehat{\operatorname{FDR}}_{\alpha}$ the FDR estimate resulting from controlling the FDR at level $\alpha$ ( $\alpha$ being classically tuned to $1 \%$ ) it is likely that

$$
\widehat{\mathrm{FDR}}_{\alpha} \leq \alpha
$$

In other words, if one cuts-off a list of putative biomarkers according to an FDR controlled at $1 \%$, the FDR estimate on this very list is likely to be slightly lower than $1 \%$. However, as the FDP remains unknown, it is the only way to safely assume that the FDP is equal to or lower than $1 \%$.

\subsection{Classical notations in proteomics}

In proteomics, most of the notions described above (see Subheading 2.1) are conflated. Since the mid-2010s, discriminating between the FDP and the FDR has progressively become standard. However, distinction between FDR (as equal to $\mathbb{E}[\mathrm{FDP}]), \overline{\mathrm{FDR}}, \widehat{\mathrm{FDR}}_{\alpha}$, and $\alpha$ is scarce. The reason is obvious: except for specific methodological publications, most of them are not useful to the community. Indeed, in practice, a proteomic researcher only needs to manipulate $\alpha$, the cut-off parameter, and to understand that after applying the FDR control accordingly, the FDP is not necessarily strictly equal to $\alpha$, but possibly slightly smaller. However, the everyday language is error-prone: when one says or writes "We selected the putative biomarkers at an FDR of $1 \%$," what is referred to as FDR is not $\mathbb{E}[\mathrm{FDP}]$, 
$\widehat{\mathrm{FDR}}$, or $\widehat{\mathrm{FDR}}_{\alpha}$, but $\alpha$.

To cope with this, it is possible to rely on other notations. They are not as formal as those of mainstream biostatistics (see Subheading 2.1) although they are sometimes reported in mathematics works [4]. However, they are sufficient for a rigorous everyday work in a proteomic lab. Essentially, it amounts to conflate the FDR estimate with $\alpha$, and to define the FDR control as a procedure which provides the following guarantee with a sufficiently high probability:

$$
\mathrm{FDR} \geq \mathbb{E}[\mathrm{FDP}]
$$

This formulation can be misleading in the sense it gives the impression that the FDR control procedure indeed controls the FDP (see Note 3). However, it has two advantages: First, it makes the everyday language compliant with the minimum amount of statistical notions possible; second, it simplifies the understanding of other statistical notions such as "q-value" or "adjusted p-value," as using this formalism, they are simply equivalent to the FDR, as detailed in [5]. In the rest of the protocol, the naming conventions resulting from Eq. 1 are used, so that FDR refers to $\alpha$, the FDR level tuned by the practitioner to perform FDR control.

\subsection{Other notations used in this protocol}

Hereafter, the following mathematical notations are used:

1. $n$ : the number of biological samples.

2. $p$ : the number of proteins to include in differential analysis.

3. $X \in \mathbb{R}^{n \times p}$ : the matrix of protein abundances, where each row corresponds to a sample and each column corresponds to a protein.

4. $X_{j}$ : the vector of abundance of the $j$-th protein, i.e. the $j$-th column of $X$.

5. $x_{i, j}$ : the abundance value of $j$-th protein for the $i$-th replicate.

6. $y$ : the vector representing the condition label (numerical value) of biological samples, of length $n$. For example, the $i$-th coefficient of $y$ is 1 if the $i$-th sample comes from the healthy condition, and -1 if it comes from the disease condition.

7. $X^{\mathrm{Ko}} \in \mathbb{R}^{n \times p}$ : the knockoff dataset, generated from original dataset matrix $X$.

8. $X_{j}^{\mathrm{Ko}}$ : the knockoff vector of abundance of the $j$-th protein.

9. $W$ : the vector of scores of all proteins (only the original ones, not the knockoff), of length $p$.

10. $W_{j}$ : the score associated to the $j$-th protein. A large positive value $W_{j}$ is evidence that the protein $j$ is differentially expressed. It is typically constructed by comparing the predictive power of $X_{j}$ and $X_{j}^{\mathrm{Ko}}$ of the sample conditions. Swapping $X_{j}$ and $X_{j}^{\mathrm{Ko}}$ should swap the sign of $W_{j}$. A null $W_{j}$ means that both $X_{j}^{\mathrm{Ko}}$ and $X_{j}^{\mathrm{Ko}}$ bring the same amount (or lack thereof) of information on the condition. 


\section{Material}

\subsection{R version}

$\mathrm{R}$ version 4.0.3 (or above) is required to use the following packages. We recommend using an integrated development environment like Rstudio to execute the commands of this protocol. It can be downloaded from https://www.rstudio.com/.

\subsection{Packages}

The following packages are necessary:

1. The packages knockoff, lars ([13]), and glmnet ([11]) must be installed from the CRAN:

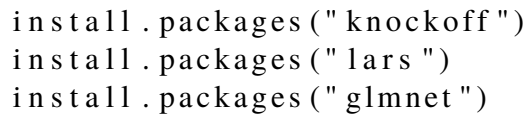

2. $\mathrm{cp} 4 \mathrm{p}$ [12] provides two datasets with controlled ground truth: They result from analysis of samples containing different abundance of 48 human proteins spiked in a yeast background [19]. The p-values from a Welch $t$-test associated to each protein are also provided, along with functions to apply Benjamini-Hochberg procedure for differential analysis. To install $\mathrm{cp} 4 \mathrm{p}$ package, it is first necessary to install the BioConductor [15] packages it depends on:

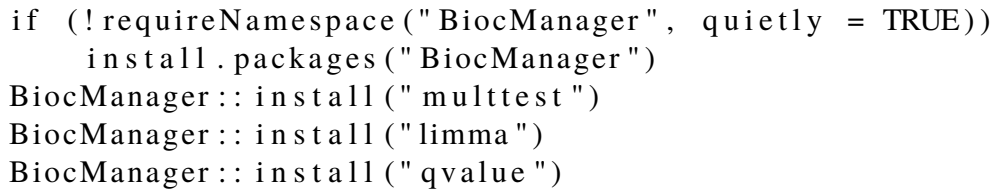

3. Then $\mathrm{cp} 4 \mathrm{p}$ can be installed from the CRAN:

in stal1 .packages ("cp4p")

4. Finally, load the packages in the environment:

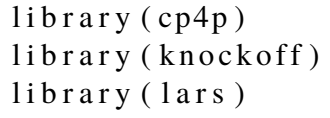

\subsection{Data Format}

This protocol relies on a data format which is quite uncommon in proteomics (see Note 4). The input data $X$ on which FDR control is applied should have at least 3 rows, i.e. at least biological 3 samples in total are needed. The number of proteins to include in differential analysis can be arbitrary. Values of abundance in $X$ should be $\log _{2}$-scaled.

For conveniency, we use two datasets in this protocol: A dataset resulting from real mass-spectrometry output, called LFQRatio25 (see Subheading 3.4), and a simulated dataset with adjustable parameters (see Subheading 3.5). 


\subsection{Data loading from cp4p}

The following commands enable to load and prepare LFQRatio25 dataset [12]:

1. Load the dataset with the following command:

data (" LFQRatio25 ")

2. Then, abundances values for all 6 samples are extracted to form the rows of the X_yups variable:

$$
\text { X_yups = } \mathrm{t}(\text { LFQRatio25 }[, 1: 6])
$$

3. Similarly, vector y_yups contains the condition labels of these samples:

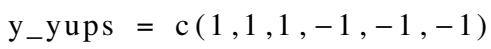

4. For this particular dataset, differentially abundant proteins (or in statistical language, variables under the alternative hypothesis $H_{1}$ ) are known. It is possible to display their name and their index in the list of proteins. These are the 46 first proteins, as the output of this code chunk suggests (see Note 5):

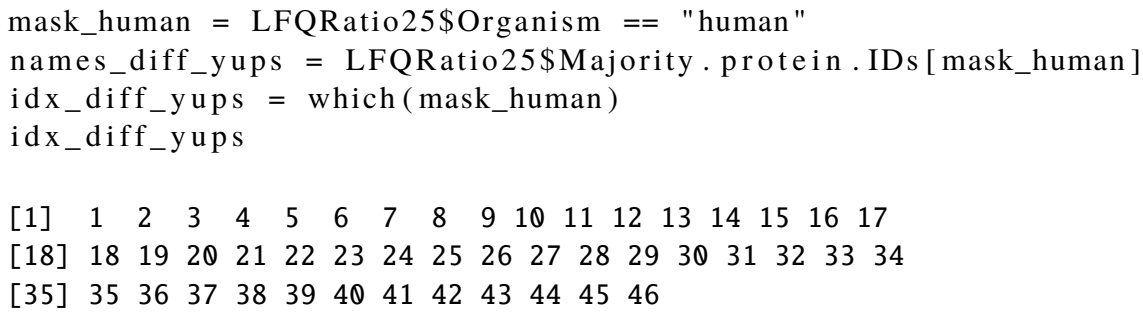

5. Check the dataset to make sure the same dataset is obtained:

head (X_yups $[, 1: 5])$

$$
[, 1] \quad[, 2] \quad[, 3] \quad[, 4] \quad[, 5]
$$

$\begin{array}{lllllll}\text { A.R1 } & 31.27392 & 29.48101 & 29.80982 & 29.10410 & 26.85626\end{array}$

$\begin{array}{lllllll}\text { A.R2 } & 31.27147 \quad 29.46032 & 29.84163 & 29.22384 & 27.11535\end{array}$

A.R3 $31.26327 \quad 29.45797 \quad 29.83771 \quad 29.00945 \quad 26.94358$

B.R1 29.83022 $28.04973 \quad 28.41002 \quad 27.45505 \quad 25.71735$

B.R2 $29.8141328 .02686 \quad 28.38101 \quad 27.58463 \quad 25.74196$

B.R3 29.84867 28.00774 28.42514 27.52028 24.62264

\subsection{Data simulation}

The following commands enable to prepare a simulated dataset:

1. The code below randomly generates a dataset broadly akin to LFQRatio25. Due to randomness, it will be different from one run to another. To ensure the results are reproducible and to obtain same results as in the remaining of the protocol, use the following optional command to set the random seed (see Note 6): 
set. seed (1234)

2. Tune the parameters of the dataset:

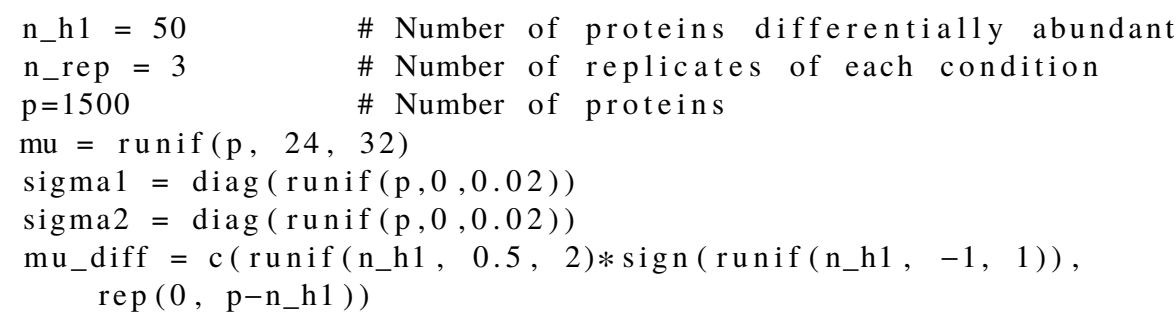

3. Create and concatenate arrays of both conditions:

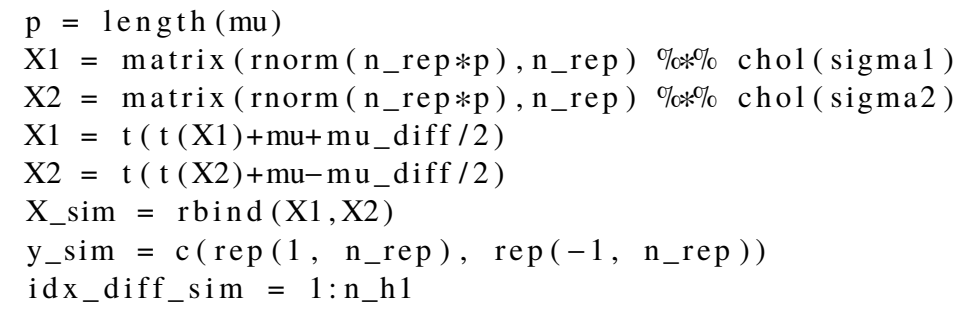

4. Check the dataset to make sure there are no mistakes:

head (X_sim $[, 1: 5])$

$$
[, 1] \quad[, 2] \quad[, 3] \quad[, 4] \quad[, 5]
$$

$[1] \quad 23.9651928 .55181 \quad 27.95301 \quad 29.64470 \quad$,

$\left[\begin{array}{llllll}2,] & 24.04396 & 28.21652 & 27.74679 & 29.56570 & 31.51248\end{array}\right.$

$\left[\begin{array}{lllllll}3,] & 24.05717 & 28.39634 & 27.90406 & 29.74762 & 31.56869\end{array}\right.$

$\left[\begin{array}{lllllll}4,] & 25.65308 & 29.55612 & 29.92890 & 28.21821 & 30.47228\end{array}\right.$

$\left[\begin{array}{lllllll}5,] & 25.74846 & 29.63377 & 29.77653 & 28.30777 & 30.32441\end{array}\right.$

$\left[\begin{array}{llllll}6,] & 25.89306 & 29.58248 & 29.80624 & 28.33325 & 30.52107\end{array}\right.$

\section{Methods}

This section falls into the following subsections:

1. We explain how to apply the original knockoff-filter procedure to control the FDR for differential expression analysis. Precisely, we show how to (1) generate knockoff variables; (2) compute a score for each protein/knockoff pair; (3) select differentially abundant proteins for a predefined target FDR.

2. We detail how to replace the default scoring strategy with other ones, and compare these alternative knockoff procedures to the classical BenjaminiHochberg $(\mathrm{BH})$ procedure.

3. We propose some code to illustrate the sensitivity of the knockoff filter procedure to the random generation of knockoffs. 


\subsection{Original knockoff procedure}

1. Choose the dataset on which applying the knockoff procedure:

(a) To apply it on the LFQRatio25 dataset, use:

$$
\begin{aligned}
& X_{-} \text {data }=X_{-} \text {yups } \\
& \text { y_data }=\text { y_yups } \\
& \text { idx_diff }=\text { idx_diff_yups }
\end{aligned}
$$

(b) Alternatively, to apply it on the simulated dataset, use:

$$
\begin{aligned}
& \text { X_data }=\text { X_sim } \\
& \text { y_data }=y_{-} \text {sim } \\
& \text { idx_diff }=\text { idx_diff_sim }
\end{aligned}
$$

For the rest of this section, we will use the LFQRatio25 dataset.

2. Rescale the data to have null mean and unitary variance for each protein abundance vector (i.e. for each $X_{j}$ ) (see Note 7):

$X_{-}$data $=$scale $\left(X_{-}\right.$data $)$

3. Execute these commands to generate the knockoff dataset from original data with a fixed seed (see Note 8):

set. seed (1234)

X_data_k = create.second_order $\left(X_{-}\right.$data $)$

4. For each protein, compute a score based on the Lasso path of covariates (see Note 9). An inevitable warning concerning the lack of replicates appears: "one multinomial or binomial class has fewer than 8 observations; dangerous ground."

set. seed (1234)

W_lasso = stat.lasso_lambdasmax_bin $\left(X_{-}\right.$data, X_data_k, y_data)

5. Set the value of targeted FDR, compute the resulting threshold, and select proteins for which their score is above this threshold. The target_fdr parameter must be a number between 0 and 1 . The offset parameter determines which FDR estimator to use, it can be set to either 0 or 1 (see Note 10). When offset is 0 , a biased FDR estimate is used, and when offset is 1, a non-biased, yet more conservative estimate is used.

target_fdr $=0.05$

thres $=$ knockoff.threshold $\left(W_{-}\right.$lasso, fdr=target_fdr, offset $\left.=0\right)$ selected_lasso $=$ which $\left(W_{-}\right.$lasso $>=$thres $)$

6. This step and the following ones are optional, as they can only be applied for a dataset endowed with a ground truth, such as LFQRatio25 or a simulated dataset. Display the names of proteins selected as differentially abundant at the FDR tuned with the target_fdr parameter (here 0.05).

names_diff_yups[selected_lasso] 
[1] PQ2768upsedyp ALBU_HUMAN_upsedyp - CON__PQ2768-1

[2] O00762upsedyp UBE2C_HUMAN_upsedyp

[3] P00709upsedyp LALBA_HUMAN_upsedyp

[4] PQ2788upsedyp TRFL_HUMAN_upsedyp

[5] P06396upsedyp GELS_HUMAN_upsedyp

[6] P12081upsedyp SYHC_HUMAN_upsedyp

7. This code instantiates useful functions to compute the FDP and power from ground truth data. For a certain selection level $\alpha$, the power is defined as

$$
\text { Power }_{\alpha}=\frac{\# \text { of selected original variables under } H_{1}}{\# \text { of original variables under } H_{1}} .
$$

Where $H_{1}$ denotes the alternative hypothesis, i.e. "the protein is differentially abundant." The power gives a measure of how well our selection covers all the proteins differentially expressed:

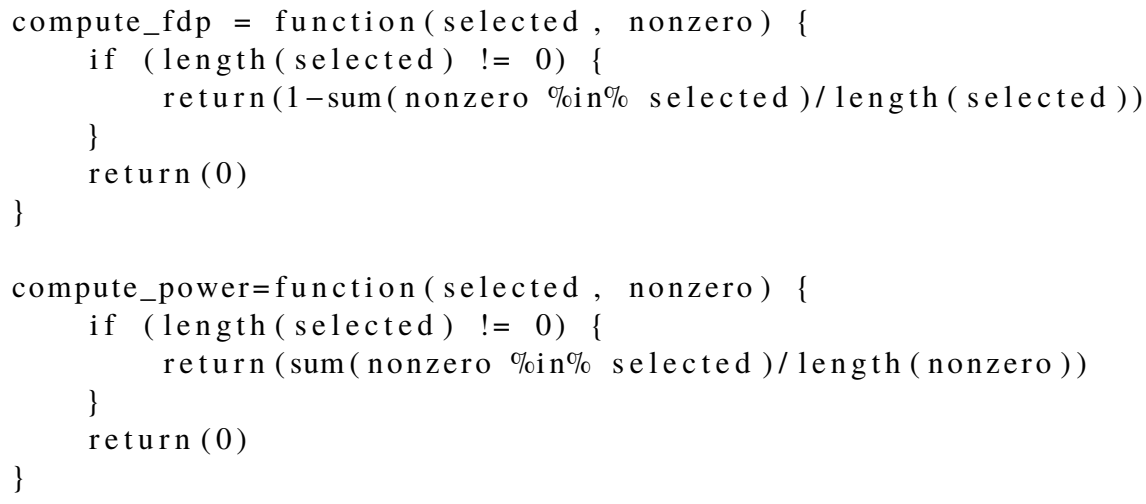

8. The following code computes the FDP and power of the procedure for a user-defined range of target FDRs (for both offset values):

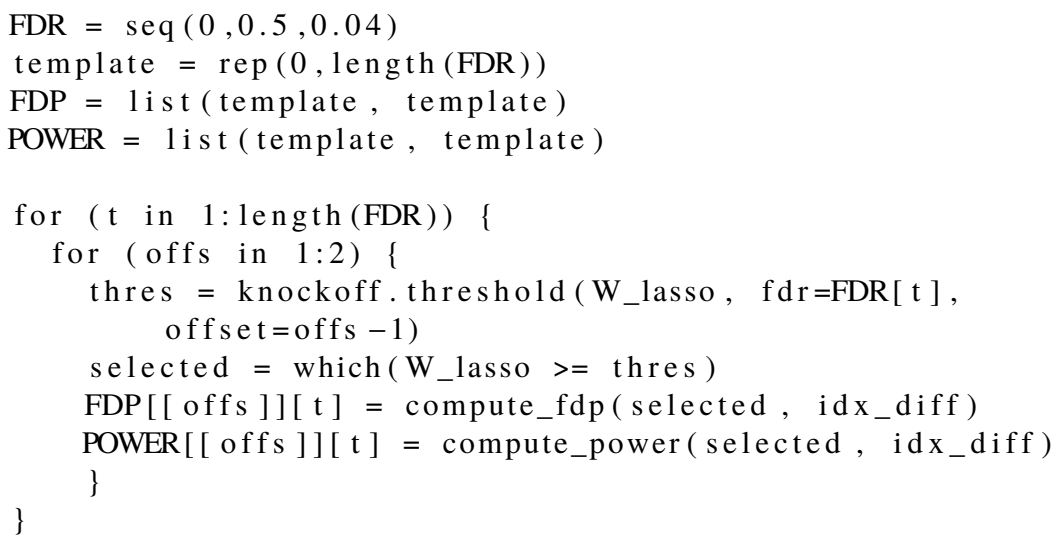

9. Using the results computed at the previous step, the following code displays the FDP and power as a function of the FDR (see Figure 1 for LFQRatio2 5 and Figure 2 for simulated dataset): 

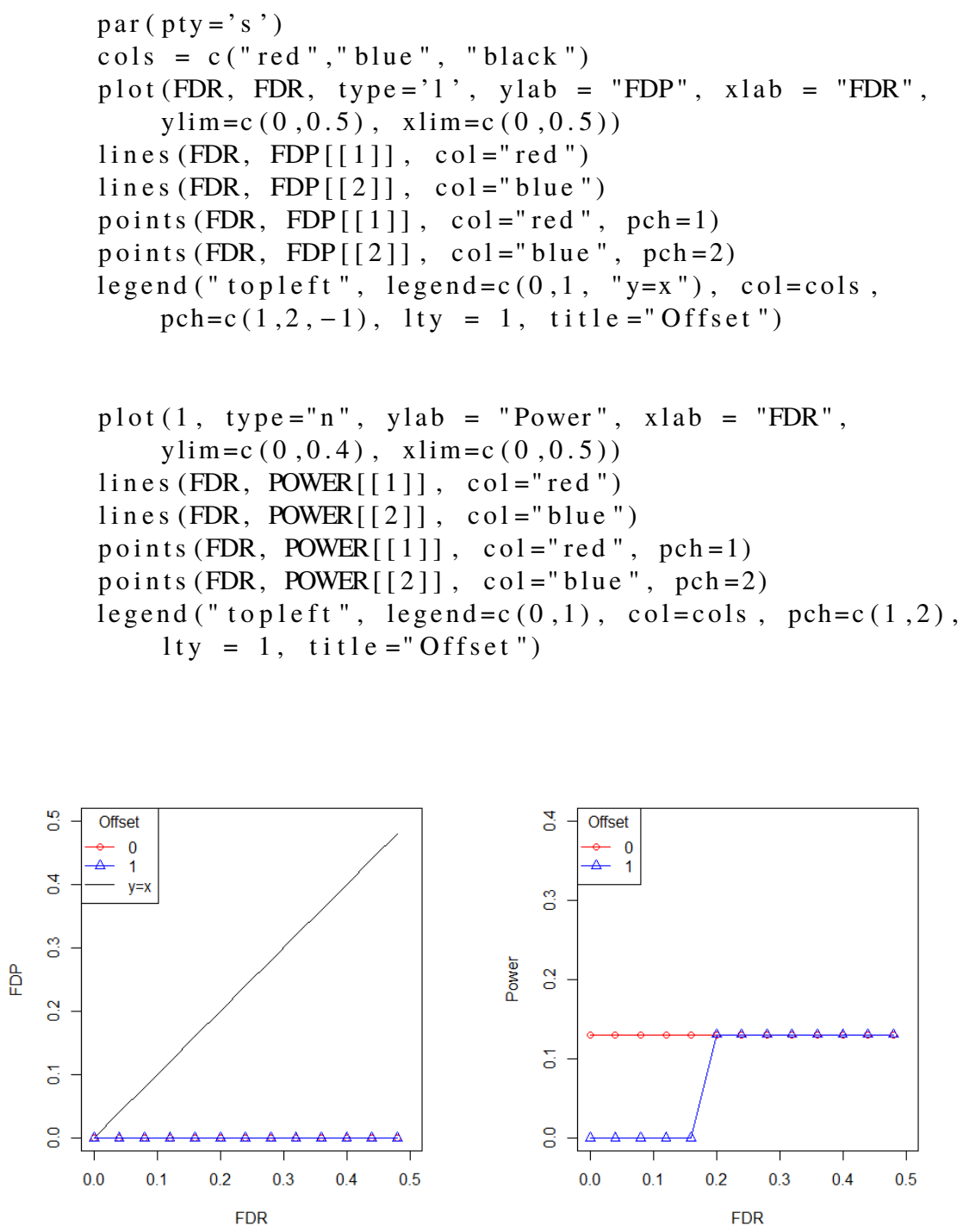

Figure 1: FDP and power vs. FDR for LFQRatio25 dataset, with and without offset, for the knockoff filter procedure with Lasso-based scores.

We notice that FDP and power curves on Figures 1 and 2 are almost always horizontal. This means that variables selected remain the same whatever the FDR target chosen. When the offset equates 1 (unbiased estimator), no proteins are deemed differentially expressed below a certain value of FDR. Thus, even though their are no false positive, there are no true positive either, making the FDR control through knockoff filters practically useless.

We mainly explain this over-conservativeness by the usage of variable selection with the Lasso algorithm, at the step of $W$ scores computation. In fact, in the setting 

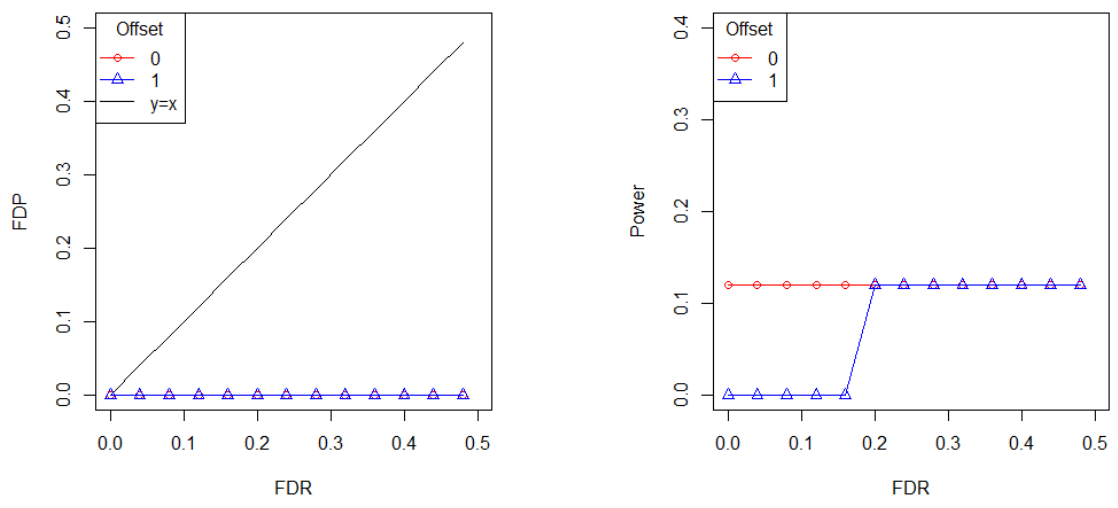

Figure 2: FDP and Power vs. target FDR for the simulated dataset, with and without offset, for knockoff procedure with Lasso-based scores.

$n<<p$, the Lasso algorithm will only select $n$ variables. This is problematic for differential expression analysis where the total number of samples rarely exceeds the number of a priori differentially expressed proteins. On top of that, as very few covariates are selected, and some original variables are much more differentially abundant than all the others, knockoff variables are almost never selected. Thus, estimating the number of false discoveries from the number of selected knockoffs is not appropriate in our cases. These efficiency of variable selection with Lasso is thoroughly discussed in [21].

\subsection{Scoring methods based on forward stagewise regres- sion and $t$-test}

Preliminary experimental comparisons highlighted the knockoff procedure accuracy highly depends on the chosen feature selection algorithm. We herefater describe two procedures that we found to address the issue described above (see Subheading 4.1). The first scoring method consists in using forward stagewise selection (FS) algorithm (see Note 11). The second one is derived from the variable selection procedure classically used in proteomics: it amounts to computing a $t$-test $\mathrm{p}$-value for both original and knockoff variables; then, the final score (i.e., $\left.W_{j}\right)$ is defined by the log difference of p-values (LDP) obtained between each original variable and its knockoff.

1. To instantiate the functions that compute the $W_{i}$ 's for the FS and LDP methods, use the following chunks of code (it is advised to run them both, so as to allow subsequent comparisons):

(a) For the FS method:

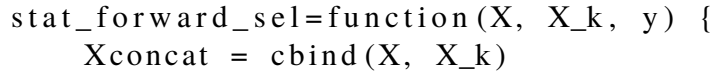




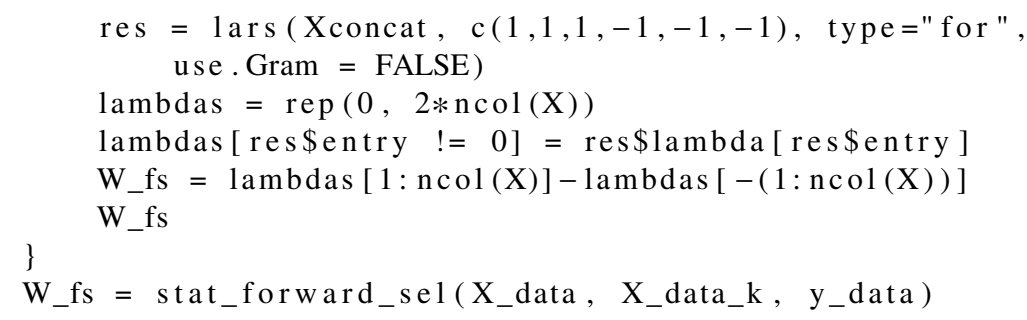

(b) For the LDP method:

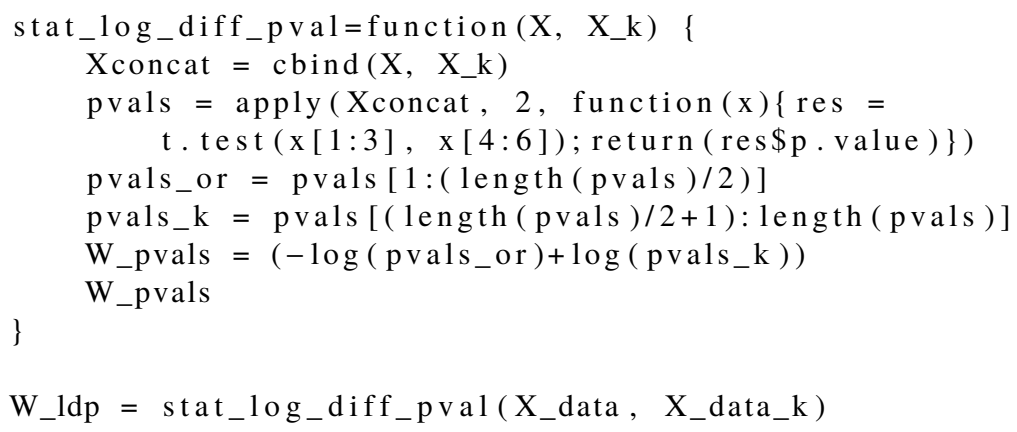

2. Plot the histogram of $W_{i}$ 's to better visualize the selection process (see Figure 3 for LFQRatio25 dataset):

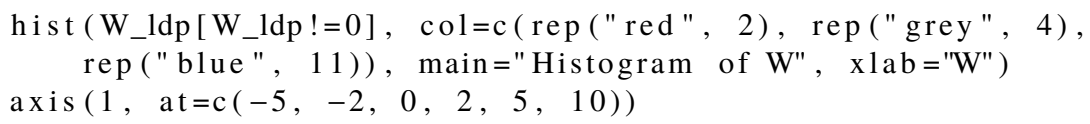

3. To illustrate the interest of using FS and LDP within the knockoff filter procedure, we compare those two approaches with the classically used BenjaminiHochberg (BH) procedure. Depending on the dataset being LFQRatio25 or the simulated one, the code differs:

(a) With LFQRatio25, the p-values resulting from Welch $t$-test are provided in the dataset:

$$
\begin{aligned}
& \text { pvals }=\text { LFQRatio25 }[, 7] \\
& \text { res }=\text { adjust.p }(\text { pvals }, \text { pi0.method = 1) }
\end{aligned}
$$

(b) With the simulated dataset, p-values must be computed beforehand (a Welch $t$-test is also used here):

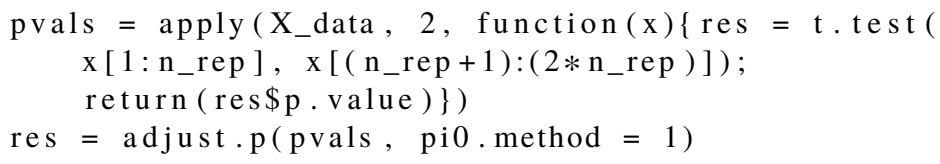

4. Compute the FDP and power for BH and knockoff filter procedure with LDP and FS methods (with offset=1), at different FDR levels:

FDP = list (template, template, template)

POWER = list (template, template, template) 


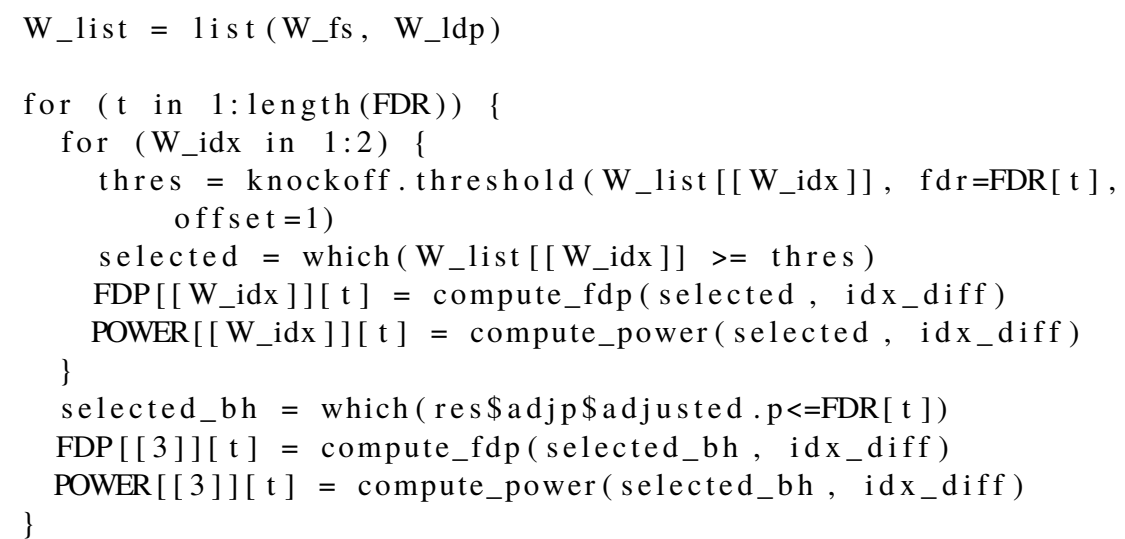

5. Finally plot the FDP and power vs. FDR level, as illustrated on Figures 4 and 5, respectively for the LFQRatio25 and simulated datasets):

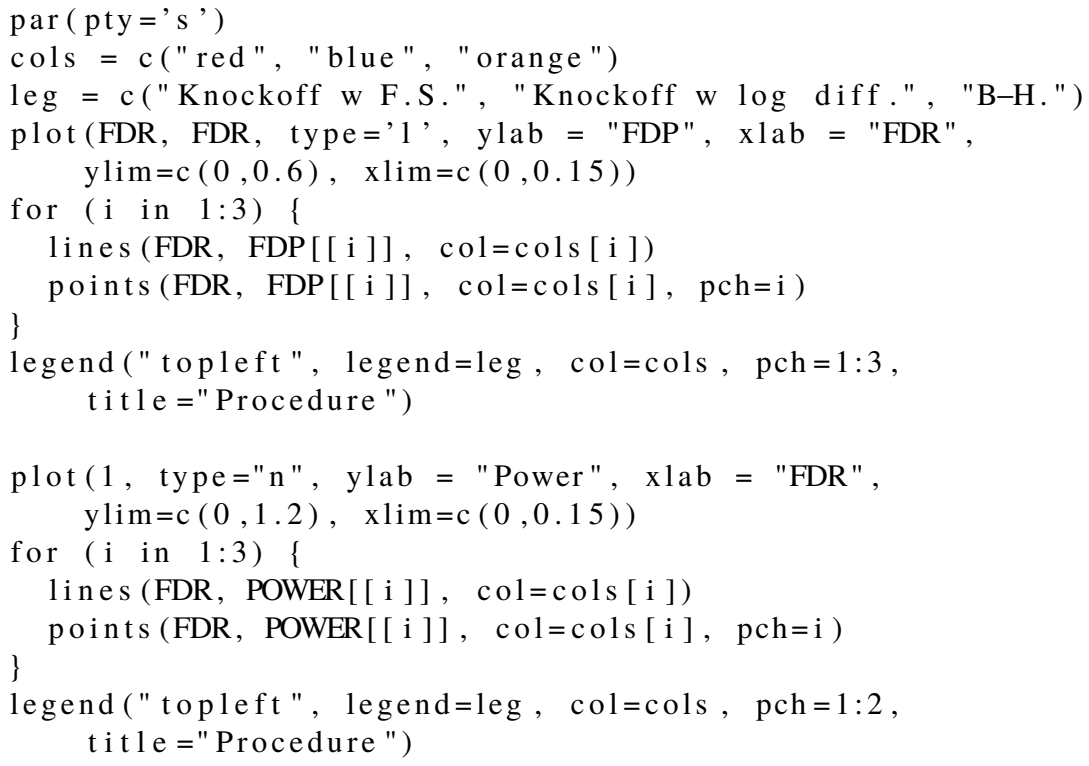

We observe that the knockoff filter procedure with LDP broadly follows the same trend as the BH one on LFQRatio25 (see Figure 4). By construction, the LDP scores is never null, yielding a rather symmetric distribution (see Figure 3). The largest positive scores (depicted in the right hand tail) result from differentially abundant proteins, while the left hand one amounts to selected knockoff proteins. The distribution being more symmetric than when using the Lasso, it is possible to select a larger subset of proteins at a given FDR. However, when using the FS based scores, knockoff filters roughly behaves as with the Lasso, yielding a greater but yet insufficient power.

Finally, the $\mathrm{BH}$ procedure also yields anti-conservative results on LFQRatio25, as the FDP is always higher than the FDR. However, this can be explained by other 


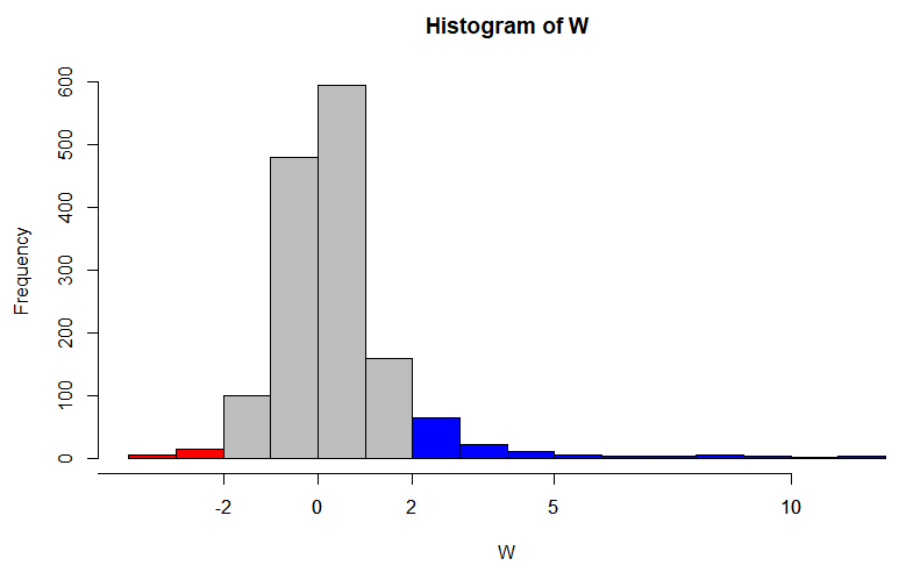

Figure 3: Histogram of scores $W_{i}$ 's obtained with log diff of p-values scoring method, on LFQRatio25 dataset. The blue area correspond to original variables that are selected, and the red area represent knockoff variables selected, both at a threshold of 2 (hence, a conservative FDR estimate at a selection threshold of 2 reads $\widehat{F D R}=\frac{\text { red area+1 }}{\text { blue area }}$ ).

preprocessing steps (match between runs, normalization, imputation, etc.) which tends to shrink the within-condition variance prior to differential analysis as well as to increase the risk of false positives that are not accounted by FDR control. Indeed, Benjamini-Hochberg is conservative on simulated data (see Figure 5).

\subsection{Sensitivity of FDR control to knockoff used}

Knockoff generation with create. second_order function (see Subheading 4.1, Step 3) involves the random draw of a knockoff matrix (similarly to the random generation of decoy sequences). Hence, on a given dataset, running two consecutive FDR control procedures with knockoff filters should lead to slightly different results. We hereafter propose several experiments to illustrate the sensitivity of the knockoff filter procedure to the knockoff generation, as well as to evaluate its magnitude.

1. Generate 30 knockoff datasets and store them in a list (depending on the machine, this step may last between 30 minutes to an hour):

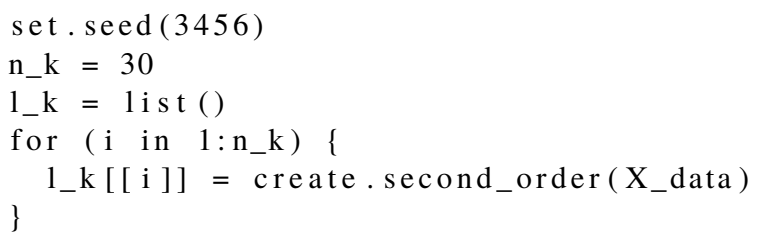

2. Apply the knockoff filter procedure to each knockoff series, with FDR varying from $1 \%$ to $15 \%$. In this example, the scoring method used is LDP. For all the knockoff series, the effective FDP $v s$. FDR curves are iteratively plotted, 

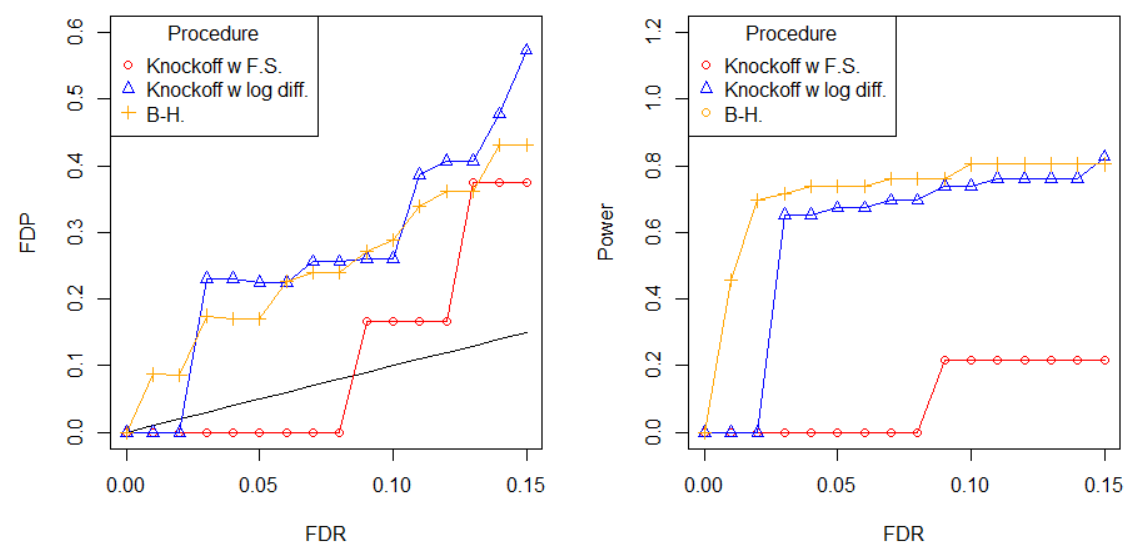

Figure 4: FDP and power vs. target FDR for knockoff filter procedure with offset=1 applied with forward stagewise selection and log diff of p-values scoring, and BenjaminiHochberg procedure, obtained with LFQRatio25.

leading to a display akin to that of Figure 6. The proteins selected at an FDR of $5 \%$ for each knockoff series are retained in a matrix referred to as scores:

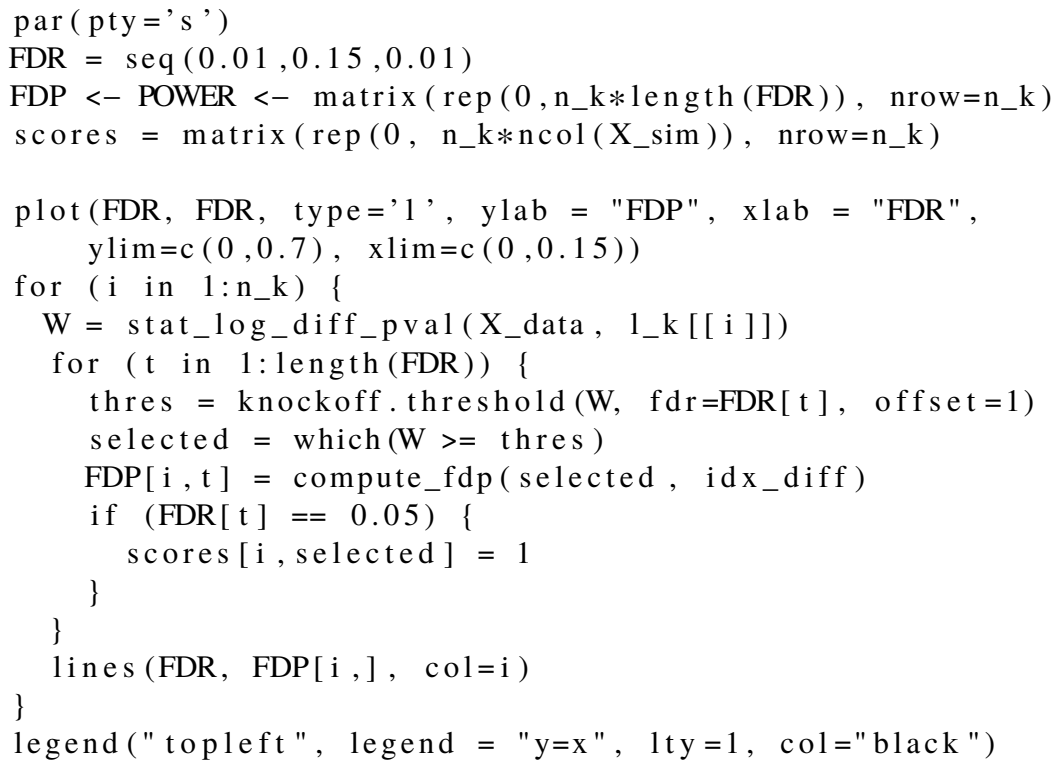

3. Finally, plot a heatmap featuring the scores matrix which highlights with different colors the selected proteins under $H_{0}$ and $H_{1}$ for each knockoff filter series, at an FDR target of 5\%. (see Figure 7): 

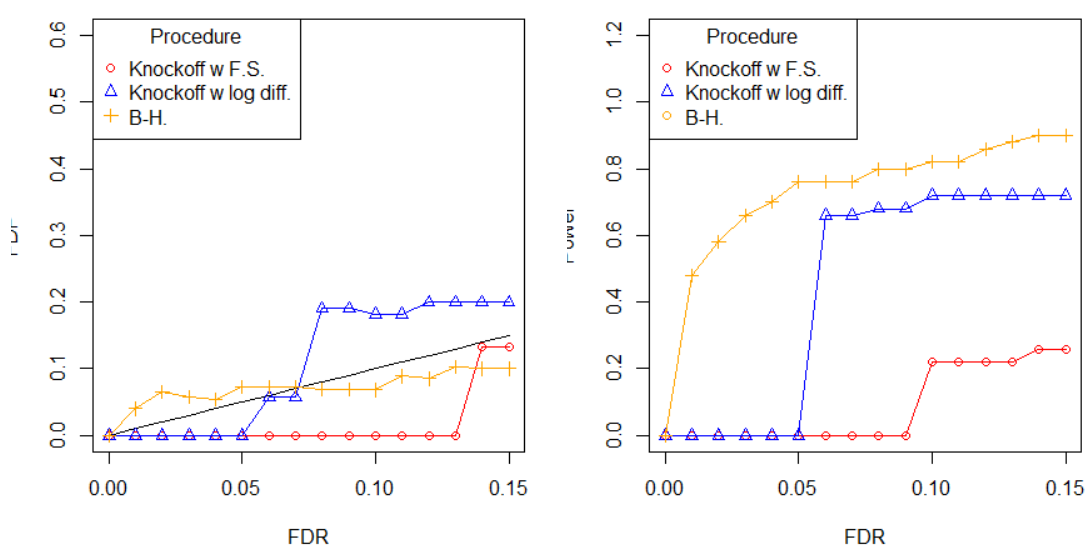

Figure 5: FDP and power vs. target FDR for knockoff procedure with offset=1 applied with forward stagewise selection and log diff of p-values scoring, and BenjaminiHochberg procedure, obtained with simulated data.

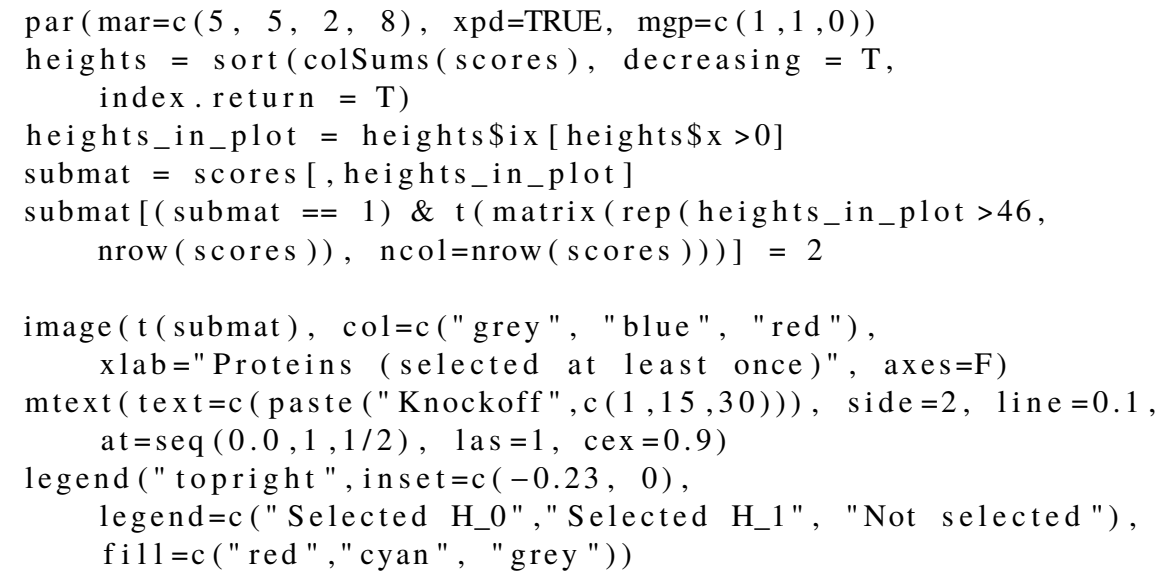

Figures 5 and 7 emphasize the important variability resulting from the random nature of knockoff filters. To counter this variability, [18] proposes a method to aggregate multiple knockoffs. In fact, similar sensitivity has already been commented upon with target-decoy procedures [17], so it seems to be a problem ubiquitous to FDR control procedures which involve simulating artifactual data under the null hypothesis. Finally these observations provide an intuitive support for the tools described in [10], which relies on multiple decoy databases to construct a knockoff-like score. 


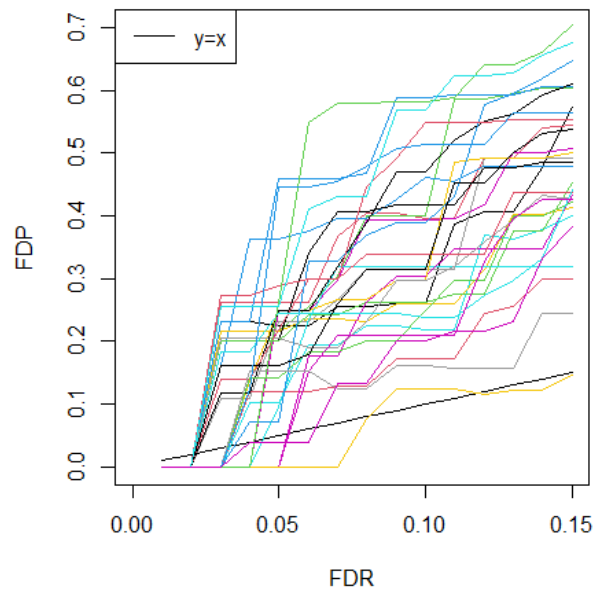

Figure 6: Curves of FDP vs. FDR for 30 different Knockoff procedure, applied with $\log$ diff of -values score on LFQRatio25 dataset.

\section{References}

[1] Rina Foygel Barber, Emmanuel J Candès, et al. Controlling the false discovery rate via knockoffs. Annals of Statistics, 43(5):2055-2085, 2015.

[2] Yoav Benjamini and Yosef Hochberg. Controlling the false discovery rate: A practical and powerful approach to multiple testing. Journal of the Royal Statistical Society. Series B (Methodological), 57(1):289-300, 1995. ISSN 00359246.

[3] Yoav Benjamini, Abba M Krieger, and Daniel Yekutieli. Adaptive linear step-up procedures that control the false discovery rate. Biometrika, 93(3): 491-507, 2006.

[4] Patricia Bouret and Fanny Bastien. Erreurs et tests statistiques (40 min), 2018.

[5] Thomas Burger. Gentle Introduction to the Statistical Foundations of False Discovery Rate in Quantitative Proteomics. Journal of Proteome Research, 17(1):12-22, 2018. ISSN 15353907.

[6] Emmanuel Candès, Yingying Fan, Lucas Janson, and Jinchi Lv. Panning for gold: 'model-x' knockoffs for high dimensional controlled variable selection. Journal of the Royal Statistical Society: Series B (Statistical Methodology), 80(3):551-577, 2018.

[7] Yohann Couté, Christophe Bruley, and Thomas Burger. Beyond target-decoy competition: stable validation of peptide and protein identifications in mass 


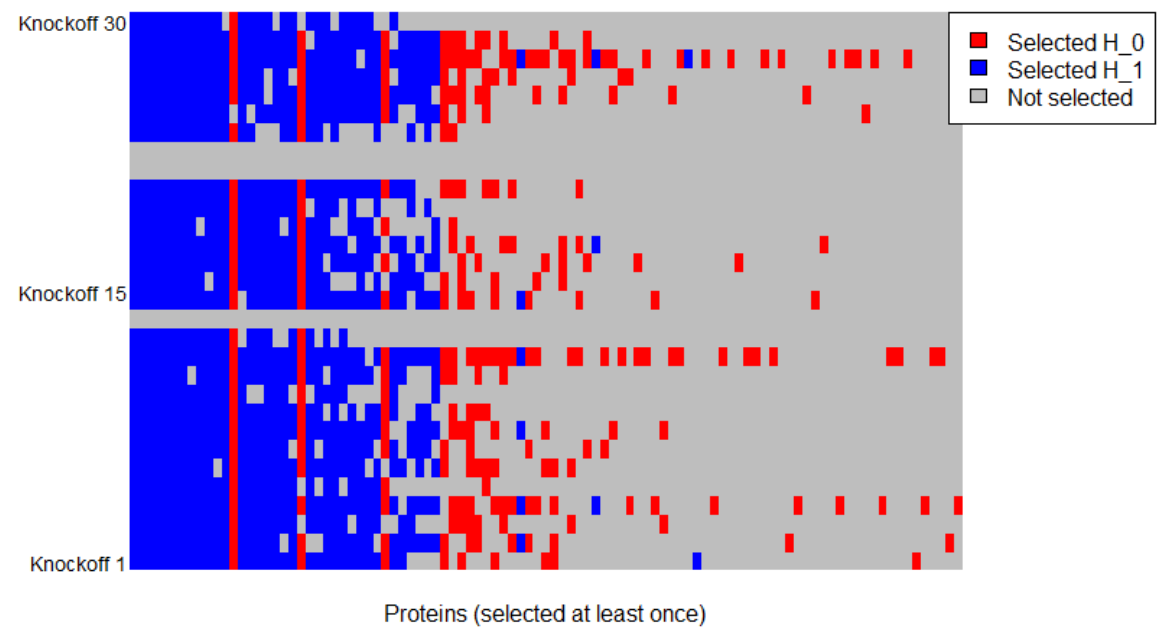

Figure 7: Proteins selected according to 30 different knockoff procedure iterations (using LDP score) on the LFQRatio25 dataset. Blue cells depict original differentially abundant proteins (human proteins) that were selected using a given knockoff. Similarly, red cells depict non-differentially abundant proteins (yeast proteins) mistakenly selected. Proteins are sorted from the most selected one (left hand side) to the least selected one (right hand side).

spectrometry-based discovery proteomics. Analytical Chemistry, 92(22): 14898-14906, 2020.

[8] Bradley Efron. Large-scale inference: empirical Bayes methods for estimation, testing, and prediction, volume 1. Cambridge University Press, 2012.

[9] Joshua E Elias and Steven P Gygi. Target-decoy search strategy for increased confidence in large-scale protein identifications by mass spectrometry. Nature methods, 4(3):207-214, 2007.

[10] Kristen Emery, Syamand Hasam, William Stafford Noble, and Uri Keich. Multiple competition-based fdr control for peptide detection. preprint, 2019.

[11] Jerome Friedman, J Hastie, and Robert Tibshirani. Regularization paths for generalized linear models via coordinate descent. Journal of Statistical Software, 33(1):1-22, 2010.

[12] Quentin Giai-Gianetto, Florence Combes, Claire Ramus, Christophe Bruley, Yohann Couté, and Thomas Burger. cp4p: Calibration Plot for Proteomics, 2019. R package version 0.3.6.

[13] Trevor Hastie and Brad Efron. LARS: Least Angle Regression, Lasso and Forward Stagewise, 2013. R package version 1.2. 
[14] Kun He, Yan Fu, Wen-Feng Zeng, Lan Luo, Hao Chi, Chao Liu, Lai-Yun Qing, Rui-Xiang Sun, and Si-Min He. A theoretical foundation of the targetdecoy search strategy for false discovery rate control in proteomics. preprint, 2015 .

[15] W. Huber, V. J. Carey, R. Gentleman, S. Anders, M. Carlson, B. S. Carvalho, H. C. Bravo, S. Davis, L. Gatto, T. Girke, R. Gottardo, F. Hahne, K. D. Hansen, R. A. Irizarry, M. Lawrence, M. I. Love, J. MacDonald, V. Obenchain, A. K. Ole's, H. Pag 'es, A. Reyes, P. Shannon, G. K. Smyth, D. Tenenbaum, L. Waldron, and M. Morgan. Orchestrating high-throughput genomic analysis with Bioconductor. Nature Methods, 12(2):115-121, 2015.

[16] Lukas Käll, John D Storey, Michael J MacCoss, and William Stafford Noble. Assigning significance to peptides identified by tandem mass spectrometry using decoy databases. Journal of proteome research, 7(01):29-34, 2008.

[17] Uri Keich, Kaipo Tamura, and William Stafford Noble. Averaging strategy to reduce variability in target-decoy estimates of false discovery rate. Journal of Proteome Research, 18(2):585-593, 2019. doi: 10.1021/acs.jproteome. $8 \mathrm{~b} 00802$.

[18] Tuan-Binh Nguyen, Jérôme-Alexis Chevalier, Bertrand Thirion, and Sylvain Arlot. Aggregation of multiple knockoffs. In International Conference on Machine Learning, pages 7283-7293. PMLR, 2020.

[19] Claire Ramus, Agnès Hovasse, Marlène Marcellin, Anne-Marie Hesse, Emmanuelle Mouton-Barbosa, David Bouyssié, Sebastian Vaca, Christine Carapito, Karima Chaoui, Christophe Bruley, Jérôme Garin, Sarah Cianférani, Myriam Ferro, Alain Van Dorssaeler, Odile Burlet-Schiltz, Christine Schaeffer, Yohann Couté, and Anne Gonzalez de Peredo. Benchmarking quantitative label-free lc-ms data processing workflows using a complex spiked proteomic standard dataset. Journal of Proteomics, 132:51-62, 2016. ISSN 1874-3919.

[20] Matthew Stephens. False discovery rates: A new deal. Biostatistics, 18(2): 275-294, 2017. ISSN 14684357.

[21] Hui Zou and Trevor Hastie. Regularization and variable selection via the elastic net. Journal of the Royal Statistical Society: Series B (Statistical Methodology), 67(2):301-320, 2005. 